\title{
Histone deacetylase SIRT6 regulates chemosensitivity in liver cancer cells via modulation of FOXO3 activity
}

\author{
JIA-QING HU ${ }^{1}$, FANG DENG ${ }^{2}$, XIAO-PING HU ${ }^{3}$, WEI ZHANG ${ }^{4}$, XIAO-CHEN ZENG $^{5}$ and XUE-FEI TIAN ${ }^{6}$ \\ Departments of ${ }^{1}$ Emergency and ${ }^{2}$ Pharmacology, The Second People's Hospital of Hunan, Changsha, Hunan 410007; \\ ${ }^{3}$ Department of Pharmacology, The Affiliated Cancer Hospital of Guangzhou Medical University, Guangzhou, \\ Guangdong 510095; Departments of ${ }^{4}$ Radiology and ${ }^{5}$ Surgery, The Second People's Hospital of Hunan; \\ ${ }^{6}$ Department of Oncology, The Second People's Hospital of Hunan, \\ Clinical Medical College of Hunan University of Chinese Medicine, \\ Changsha, Hunan 410007, P.R. China
}

Received April 12, 2018; Accepted October 3, 2018

DOI: $10.3892 / o r .2018 .6770$

\begin{abstract}
Liver cancer is the leading cause of cancer-related mortality worldwide and its incidence is increasing. Considerable effort has been made in recent decades to improve the diagnosis and treatment of liver cancer. Advanced liver cancer often exhibits a poor response to chemotherapy and poor prognosis due to acquired chemoresistance and tumor recurrence. Understanding the precise molecular mechanisms that are responsible for chemotherapeutic drug-induced cell death could potentially identify novel therapeutic targets and improve liver cancer treatment. In the present study, it was demonstrated that in response to doxorubicin, the most frequently used chemical compound for liver cancer treatment, histone deacetylase sirtuin 6 (SIRT6) is specifically downregulated. This enables forkhead box O3 (FOXO3) upregulation, translocation into the nucleus and increased expression of its target genes p27 and Bim, which further induce apoptosis. Overexpression of SIRT6, but not enzyme-inactivated mutants, prevents FOXO3 translocation into the nucleus and doxorubicin-induced cell death. SIRT6 interacts with FOXO3 and this interaction increases FOXO3 ubiquitination and decreases its stability. Finally, it was identified that the effect of SIRT6 in preventing doxorubicin-induced cell death requires FOXO3. Overexpression of SIRT6 could not prevent doxorubicin-induced cell death in FOXO3-knockdown cells. Therefore, it was concluded that SIRT6 plays a central role in determining doxorubicin-induced cell death via modulation
\end{abstract}

Correspondence to: Professor Xue-Fei Tian, Department of Oncology, The Second People's Hospital of Hunan, Clinical Medical College of Hunan University of Chinese Medicine, Changsha, Hunan 410007, P.R. China

E-mail: windsame@163.com

Key words: sirtuin, post-translational modification, apoptosis, liver cancer of FOXO3 activity. Therapeutic targeting of SIRT6 and/or FOXO3 may offer novel strategies for treatment of liver cancer.

\section{Introduction}

Liver cancer remains an important global public health issue accounting for $9.1 \%$ of all cancer-related deaths worldwide (1) due to its aggressive nature and extremely poor survival rate (2). Primary liver cancer is rare during childhood, representing $1 \%$ of all pediatric neoplasms $(3,4)$. More than $80 \%$ of all liver cancer in infant and children can be identified as hepatoblastoma, which occurs at an annual incidence of 0.05-0.15 patients per 100,000 individuals $(3,5)$. The vast majority of primary liver cancer in adults is hepatocellular carcinoma (HCC), accounting for more than $85 \%$ of all primary liver cancer cases (6). Although considerable effort has been made in recent decades to improve the diagnosis and treatment of $\mathrm{HCC}$, it remains the second leading cause of cancer-associated mortality worldwide (7). One of the most promising developments in liver cancer treatment has been targeted delivery of cytotoxic chemotherapy agents directly into tumors. Selective injection of embolizing agents, in combination with doxorubicin, into the arteries feeding tumors, or transarterial chemoembolization (TACE), has been demonstrated to provide a survival benefit in patients with unresectable liver cancer and has now become the standard treatment for patients with intermediate stage HCC (8). Unfortunately, advanced liver cancer often has a poor response to TACE and prognosis due to acquired chemoresistance and tumor recurrence. Therefore, understanding the precise molecular mechanisms underlying liver cancer chemoresistance could potentially identify novel therapeutic targets and improve the treatment of liver cancer.

Sirtuin (SIRT) proteins are a family of evolutionarily conserved $\mathrm{NAD}^{+}$-dependent deacetylases. In mammals, seven SIRT proteins have been identified, which share NAD ${ }^{+}$binding and catalytic domains, but target different substrates (9). SIRT proteins modulate numerous biological processes, including metabolism $(10,11)$, cell survival (12-14), differentiation (15), DNA repair (16) and cancer development (17). These effects are primarily achieved by deacetylating lysine 
residues on histones, transcription factors or coactivators. At present, sterol regulatory element-binding protein-1c (18), forkhead box O3 (FOXO3) (19), p53 (20) and p65 (21) are well-established SIRT targets. SIRT1 deacetylates p53 and restrains DNA damage-induced p53 acetylation, thereby suppressing cell cycle arrest and apoptosis (22). In addition, previous reports indicate that multiple SIRT family members control FOXO3-dependent apoptosis through deacetylation of FOXO3 $(13,14,19,23)$. Previous studies have also demonstrated that SIRT1 and SIRT7 proteins are involved in cancer transformation and clinical outcome through both genetic and epigenetic alterations $(15,24)$.

SIRT6, one of the SIRT proteins, plays critical roles in controlling metabolism, genomic stability, inflammation, aging and cancer progression (25-28). SIRT6-deficient animals present with early lethality due to profound abnormalities, including hypoglycemia and premature aging (27). Conditional disruption of SIRT6 in hepatocytes leads to increased glycolysis, triglyceride synthesis, reduced beta oxidation and ultimately, fatty liver formation (26). SIRT6 is reported to be a tumor suppressor through both deacetylation-dependent and -independent activity in multiple types of cancer, including pancreatic, breast, colorectal and lung cancer (29-31). However, the role and mechanistic function of SIRT6 in liver cancer remain largely unexplored. Marquardt et al (17) reported that SIRT6 mRNA is downregulated in HCC, but others observed that SIRT6 protein levels in HCC cell lines and HCC patient tissues are upregulated (32). A recent study demonstrated that SIRT6 was upregulated in patients with HCC and it serves as an anti-apoptotic factor by suppressing Bax (33), suggesting that SIRT6 may play a role in chemotherapy-induced cell death.

The aim of the present study was to investigate the role of SIRT6 in doxorubicin-induced cell death in liver cancer cell lines. It was identified that in response to doxorubicin, SIRT6 was significantly downregulated. Restorative expression of SIRT6, but not enzyme-inactivated SIRT6 mutant, abolished doxorubicin-induced cell death. It was also revealed that transcriptional factor FOXO3 serves as a target of SIRT6 in this event. In response to doxorubicin treatment, FOXO3 was rapidly activated and translocated into the nucleus, binding to its target genes Bim and p27, which further induced cell death. Overexpression of SIRT6 blocked nuclear translocation of FOXO3 and apoptosis. In the absence of FOXO3, overexpression of SIRT6 no longer prevented doxorubicin-induced cell death. The present findings present a novel mechanism that controls FOXO3 activation and revealed that SIRT6 is a pivotal regulatory factor in determining liver cancer chemosensitivity. Therapeutic strategies that inhibit SIRT6 or activate FOXO3 may offer novel options for the treatment of liver cancer.

\section{Materials and methods}

Cell culture, plasmids and transfection. HepG2, Huh7 and HeLa cells were purchased from the American Type Culture Collection (ATCC; Manassas, VA, USA) and routinely preserved in Dulbecco's modified Eagle's medium (DMEM; Invitrogen; Thermo Fisher Scientific, Inc., Waltham, MA, USA) supplemented with $10 \%$ fetal bovine serum (FBS; Invitrogen; Thermo Fisher Scientific, Inc.), $50 \mathrm{U} / \mathrm{ml}$ penicillin and
$50 \mathrm{mg} / \mathrm{ml}$ streptomycin. Transfection of cells was performed in serum-free medium (Opti-MEM, Invitrogen; Thermo Fisher Scientific, Inc.) using X-tremeGENE ${ }^{\mathrm{TM}}$ HP DNA Transfection reagent (Roche Diagnostics, Indianapolis, IN, USA) according to the manufacturer's protocol.pECE-HA-FOXO3, SIRT6 Flag and pCDNA3.1 SIRT6_H133Y plasmids were respectively provided by M. Greenberg, Eric Verdin and Katrin Chua via Addgene, Inc. (Cambridge, MA, USA). Short hairpin (sh)RNA targeting FOXO3 (MISSION shRNA plasmid DNA FOXO3; TRCN0000010335, TRCN0000235487) was purchased from Sigma-Aldrich (Merck KGaA, Darmstadt, Germany).

Antibodies and chemicals. Anti-human influenza hemagglutinin (HA) antibody (cat. no. ab9110) and anti-SIRT4 (cat. no. ab124521) were purchased from Abcam (Cambridge, MA, USA). Anti-FOXO3 (cat. no. 75D8), anti-acetylated-lysine (cat. no. 9441), anti-SIRT1 (cat. no. D1D7), anti-SIRT6 (cat. no. D8D12), anti-ubiquitin (cat. no. P4D1), anti-cleaved caspase-3 (cat. no. 9661), anti-Bim (cat. no. C34C5), anti-p27 (cat. no. D69C12), anti-p-FOXO3 S253 (cat. no. 9466) and anti-poly (ADP ribose) polymerase (PARP; cat. no. 9542) were purchased from Cell Signaling Technology, Inc. (Danvers, MA, USA). Anti-GAPDH (FL-335) was purchased from Santa Cruz Biotechnology, Inc. (Dallas, TX, USA). Anti-Flag (M2) antibody, cycloheximide (CHX) and doxorubicin hydrochloride were purchased from Sigma-Aldrich (Merck KGaA).

Immunofluorescence. For indirect immunofluorescence, cells grown on coverslips were fixed with $4 \%$ paraformaldehyde at room temperature for $5 \mathrm{~min}$ and $0.2 \%$ Triton X-100 was used for cell permeation. The coverslips were inverted and $40 \mu 1$ droplets of blocking buffer (4\% goat serum) was incubated at room temperature for $45 \mathrm{~min}$ to prevent non-specific binding. Subsequently, cells were incubated with rabbit anti-HA (dilution 1:100) or rabbit anti-SIRT6 (dilution 1:100) and mouse anti-Flag (dilution 1:1,000) for $1 \mathrm{~h}$ at room temperature. Coverslips were washed with phosphate-buffered saline (PBS), followed by incubation for $1 \mathrm{~h}$ at room temperature in the dark with Alexa Fluor 488-conjugated goat anti-rabbit IgG (dilution 1:5,000; cat. no. A27.34; Molecular Probes; Thermo Fisher Scientific, Inc.). Additionally, DAPI was added for $5 \mathrm{~min}$ at room temperature to stain nuclear DNA. Images were acquired using a Nikon Eclipse Ti microscope (Nikon Corp., Tokyo, Japan).

Reverse transcription-quantitative polymerase chain reaction $(R T-q P C R)$. Total RNA was isolated cells using the TRIzol reagent (Thermo Fisher Scientific, Inc.) followed by cDNA generation with an RNA reverse transcription kit (Applied Biosystems; Thermo Fisher Scientific, Inc.). Subsequently, a CFX96 real-time system (Bio-Rad Laboratories, Hercules, CA, USA) was used to perform qPCR. Reaction volumes of $25 \mu \mathrm{l}$ were used, containing 12.5 $\mu \mathrm{l}$ SYBR Green PCR Master Mix (Applied Biosystems; Thermo Fisher Scientific, Inc.), $10.5 \mu \mathrm{l} 1 \mu \mathrm{mol} / \mathrm{l}$ primer stock and $2 \mu \mathrm{l} \mathrm{cDNA}$ (1:10 diluted). Primer sequences are presented in Table I.

Western blot analysis. Total cell lysates prepared from cells were used for detection. Briefly, cells were washed twice with ice cold PBS and then cell lysis were made by using RIPA buffer 
Table I. Primer sequences used for reverse transcription-quantitative polymerase chain reaction and ChIP assays.

\begin{tabular}{ll}
\hline Primer & \multicolumn{1}{c}{ Sequence (5'-3') } \\
\hline SIRT1 forward & TAGCCTTGTCAGATAAGGAAGGA \\
SIRT1 reverse & ACAGCTTCACAGTCAACTTTGT \\
SIRT4 forward & GCTTTGCGTTGACTTTCAGGT \\
SIRT4 reverse & CCAATGGAGGCTTTCGAGCA \\
SIRT6 forward & CCCACGGAGTCTGGACCAT \\
SIRT6 reverse & CTCTGCCAGTTTGTCCCTG \\
GAPDH forward & GAAGGTGAAGGTCGGAGTC \\
GAPDH reverse & GAAGATGGTGATGGGATTTC \\
p27 ChIP forward & TGCGCGCTCCTAGAGCTC \\
p27 ChIP reverse & TTTCTCCCGGGTCTGCAC \\
Bim ChIP forward & AGGCTAGGGTACACTTCG \\
Bim ChIP reverse & AGGCTCGGACAGGTAAAG \\
\hline
\end{tabular}

ChIP, chromatin immunoprecipitation; SIRT, sirtuin

(50 mM Tris, $\mathrm{pH} 7.5,150 \mathrm{mM}$ sodium chloride, 1\% NP-40, $0.2 \%$ SDS, $0.5 \%$ sodium deoxycholate, $0.1 \mathrm{mM}$ EDTA and $1 \%$ protease and phosphatase inhibitors) (Sigma-Aldrich; Merck KGaA). After centrifugation at 16,000 x g for $15 \mathrm{~min}$, supernatants were collected. Cell lysates $(25 \mu \mathrm{g})$ were separated by $10 \%$ SDS-PAGE and transferred to polyvinylidene difluoride membranes (Immobilon-P membranes; EMD Millipore, Billerica, MA, USA). Membranes were blocked with blocking buffer (5\% skim milk, $0.1 \%$ Tween-20 in PBS) for $1 \mathrm{~h}$ at room temperature. Following incubation with primary antibodies $(1: 1,000)$ overnight at $4^{\circ} \mathrm{C}$, membranes were then incubated with horseradish peroxidase-conjugated secondary antibodies (cat. nos. 31460 and 31430). Thermo Fisher Scientific, Inc.). Signals were detected using the ECL Plus Western Blotting Detection system (GE Healthcare, Chicago, IL, USA) with the ODYSSEY Fc, Dual-Mode Imaging system (LI-COR Biosciences, Lincoln, NE, USA).

Chromatin immunoprecipitation (ChIP) assay. ChIP assays were performed as previously described (34). Cells were fixed, washed and harvested followed by shearing of genomic DNA using sonication. Sonicated DNA $(20 \mu \mathrm{l})$ was purified and used as input DNA control. Sheared DNA was cleared and chromatin-bound DNAs were immunoprecipitated using FOXO3a and $50 \mu \mathrm{l}$ anti-HA magnetic beads (Dynabeads M-280 Sheep anti-rabbit IgG; Invitrogen; Thermo Fisher Scientific, Inc.). The eluted DNA from the beads was precipitated and analyzed by PCR using multiple primer sets, as listed in Table I.

Immunoprecipitation. HeLa cells were seeded at $4 \times 10^{6}$ cells $/ 10-\mathrm{cm}$ plate and transiently transfected with $4 \mu \mathrm{g}$ Flag-SIRT6. At 1 day after transfection, cells were lysed with RIPA buffer as described above. Cell extracts (400 $\mu \mathrm{g})$ were subjected to immunoprecipitation with $50 \mu 1$ anti-Flag M2 magnetic beads (Sigma-Aldrich; Merck KGaA) in each experiment. Western blotting was used to analyze immune complexes.
Caspase-3/-7 activity and TUNEL assay. Caspase-3/-7 activity was measured using the Caspase-Glo 3/7 Assay System (Promega Corp., Madison, WI, USA), according to the manufacturer's protocol. For TUNEL assays, at room temperature, cells were fixed with $4 \%$ paraformaldehyde for $10 \mathrm{~min}$ and then rinsed with PBS. TUNEL staining was performed using the DeadEND Fluorometric TUNEL system (Promega Corp.) according to the manufacturer's protocol. Five or more randomly selected fields were examined for quantification of TUNEL staining.

Statistical analysis. Three biological replicates and two technical repetitions were performed for each assay unless indicated otherwise. Representative results are presented. Differences between two groups were calculated using a two-tailed unpaired Student's t-test. Statistical significance among multiple groups was calculated using one-way ANOVA followed by Turkey's test. Variance between groups met the assumptions or the appropriate test. Unless otherwise stated, a P-value of $<0.05$ was considered statistically significant.

\section{Results}

Downregulation of SIRT6 following doxorubicin treatment of liver cancer cells. In order to examine the effects of doxorubicin treatment on SIRT family member proteins, HepG2 cells were treated with $1 \mu \mathrm{M}$ doxorubicin, and then mRNA and protein expression was measured at various time-points. Doxorubicin treatment resulted in significant increases in SIRT1 and SIRT4 mRNA expression and downregulation of SIRT6 mRNA level by $36 \mathrm{~h}$ (Fig. 1A). Western blot analysis indicated that there was no change in SIRT1 protein level following treatment and SIRT level was increased from $12 \mathrm{~h}$ post-treatment. Consistent with the mRNA results, SIRT6 protein was also significantly reduced $12 \mathrm{~h}$ after treatment and this effect lasted at least 36 h (Fig. 1B). Similarly, it was also observed that doxorubicin induced a significant decrease in SIRT6 protein expression in Huh7 cells (Fig. 1B). Immunofluorescence was performed to evaluate the cellular localization of SIRT6 in untreated and treated cells. The results indicated that SIRT6 protein localized to both the cytosol and nucleus in untreated cells. Consistent with the western blotting results, it was identified that doxorubicin decreased global SIRT6 intensity from $24 \mathrm{~h}$ post-treatment (Fig. 1C). These data indicated that doxorubicin treatment downregulates SIRT6 mRNA and protein levels in liver cancer cells. The inconsistencies between SIRT1 mRNA and protein levels also suggested that there may be post-transcriptional regulation following doxorubicin treatment.

To determine whether downregulation of SIRT6 is required for doxorubicin to cause liver cancer cell apoptosis, HepG2 cells were first treated with different concentrations of doxorubicin. A dose-dependent activation of caspase-3/-7 was observed after $36 \mathrm{~h}$ of doxorubicin treatment (Fig. 2A). Western blot analysis was performed to evaluate apoptotic signals by measuring PARP and caspase-3 cleavage, and it was identified found that $1 \mu \mathrm{M}$ doxorubicin caused a significant increase in cleaved PARP and caspase-3 (Fig. 2B). Therefore, this concentration was used for subsequent experiments (Fig. 2B). A TUNEL assay indicated that $1 \mu \mathrm{M}$ doxorubicin induced 

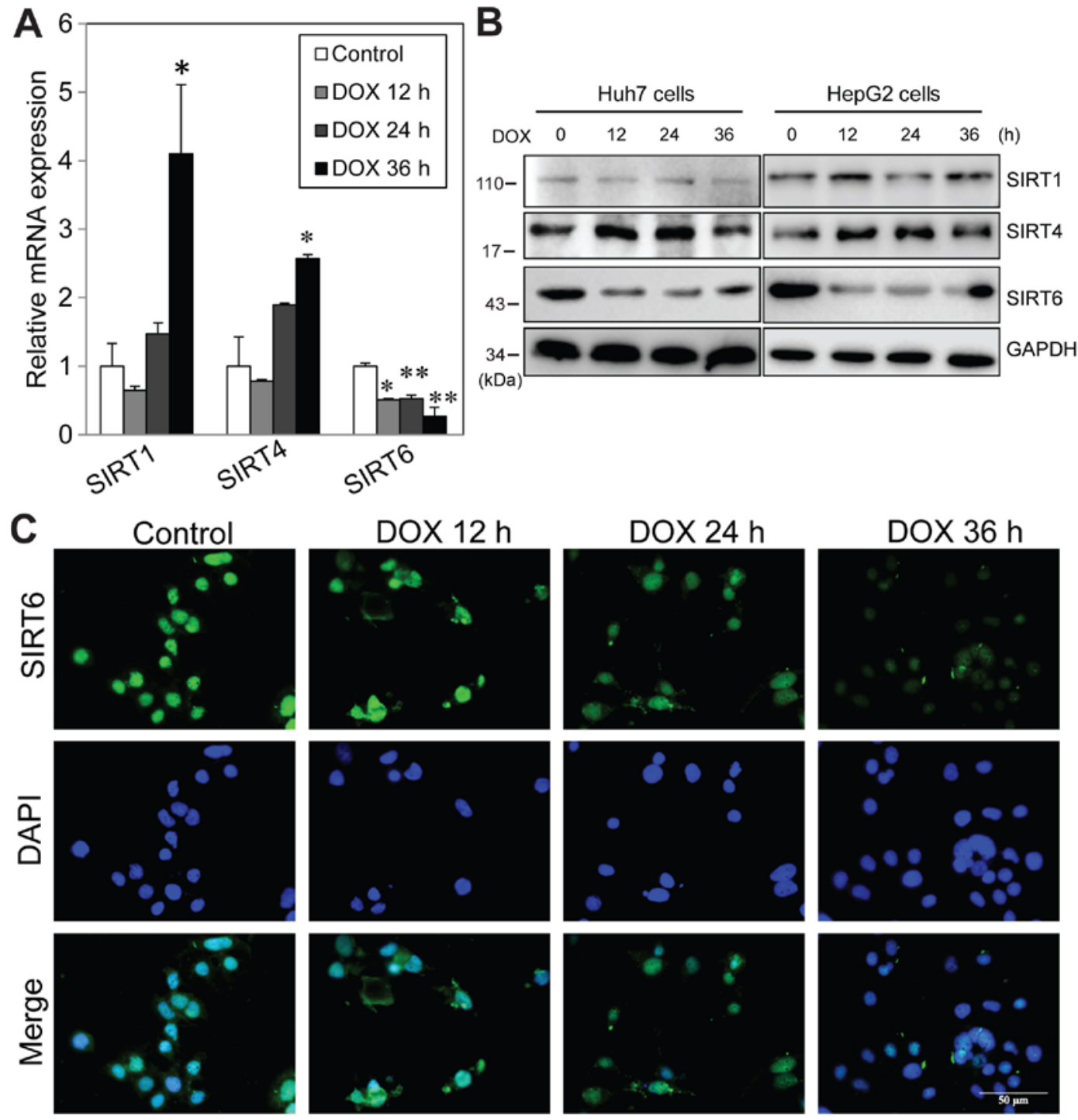

Figure 1. Doxorubicin (DOX) treatment specifically decreases SIRT6. (A) HepG2 cells were treated with $1 \mu \mathrm{M}$ DOX for $36 \mathrm{~h}$. Cells were harvested at various times as indicated. SIRT1, SIRT4 and SIRT6 mRNA levels were evaluated by reverse transcription-quantitative polymerase chain reaction. (B) HepG2 and Huh7 cells were treated with $1 \mu \mathrm{M}$ DOX for various times as indicated. Protein levels of SIRT1, SIRT4 and SIRT6 were evaluated by western blot analysis. GAPDH was used as a loading control. (C) Immunofluorescence staining of HepG2 cells that were either untreated (Control) or treated with DOX for various times as indicated by using SIRT6 antibody (green) and DAPI staining (blue). Data are presented as the mean \pm standard error of the mean of three independent experiments with technical duplicates. ${ }^{*} \mathrm{P}<0.05,{ }^{* *} \mathrm{P}<0.01$ vs. control; one-way ANOVA. SIRT, sirtuin.

a significant increase in TUNEL-positive cells from $24 \mathrm{~h}$ post-treatment (Fig. 2C). Then, Flag-tagged SIRT6 and inactive SIRT6 H133Y mutant were overexpressed in Huh7 cells followed by treatment with $1 \mu \mathrm{M}$ doxorubicin. In the absence of doxorubicin, overexpression of SIRT6 and H133Y mutant exerted no effects on PARP cleavage and caspase-3 activation. However, in the presence of doxorubicin, SIRT6 overexpression almost completely abolished doxorubicin-induced PARP and caspase-3 cleavage (Fig. 2D). A TUNEL assay indicated that restorative expression of SIRT6, but not inactive SIRT6, blocked doxorubicin-induced cell death (Fig. 2E). These data indicated that SIRT6 plays a critical role in determining cell fate in response to doxorubicin but this effect requires SIRT6 deacetylase enzyme activity.

Doxorubicin actives FOXO3, which triggers pro-apoptotic target gene expression. To determine the potential SIRT6 target that is responsible for regulating doxorubicin-induced apoptosis, multiple proteins that are known to induce apoptosis, including p53 (35), FOXO3 (36), FOXO1 (37) and Bax (33), were evaluated. It was identified that in response to doxorubicin treatment, there were no changes in FOXO1 and p53 protein expression. Bax expression was increased at $36 \mathrm{~h}$ (Fig. 3A). FOXO3 protein was significantly elevated following doxorubicin treatment (Fig. 3A). Immunofluorescence staining indicated that in both Huh7 and HepG2 cells, FOXO3 localized to both the cytosol and nucleus in untreated cells. In response to doxorubicin, FOXO3 intensity was increased and at $24 \mathrm{~h}$ post-treatment, it was primarily localized in the nucleus (Fig. 3B). In order to investigate whether nuclear FOXO3 was functionally responsible for apoptosis, a ChIP assay was performed to assess the promoter binding of FOXO3 to its target genes, which are responsible for cell cycle arrest and apoptosis, following doxorubicin treatment. 
A

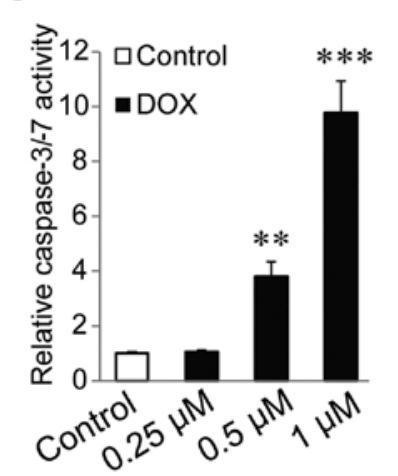

D

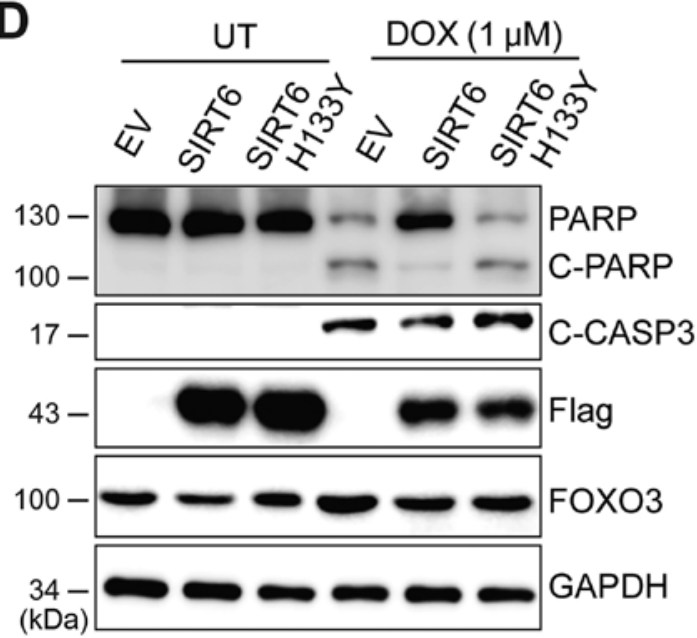

B

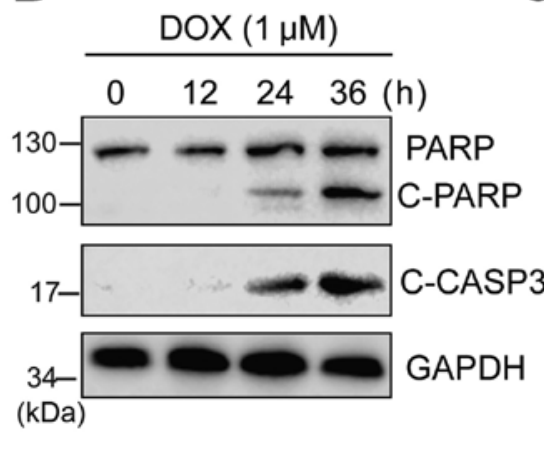

C

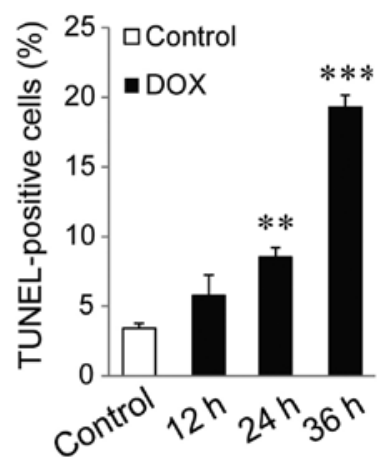

E

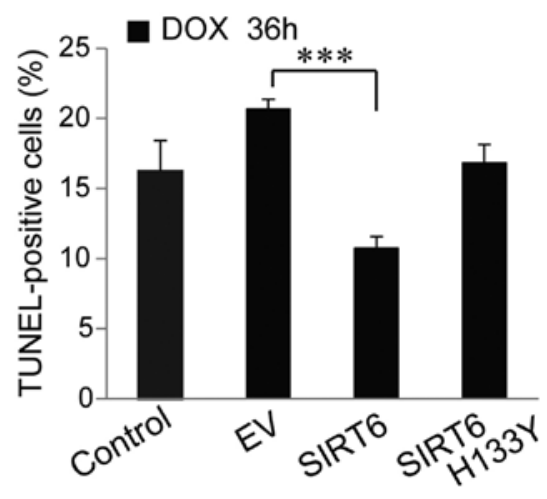

Figure 2. Doxorubicin (DOX)-induced SIRT6 downregulation is required for apoptosis. (A) HepG2 cells were treated with varies dose of DOX and caspase-3/-7 activity was examined at $36 \mathrm{~h}$ after treatment. (B and C) HepG2 cells were treated with $1 \mu \mathrm{M}$ DOX for $36 \mathrm{~h}$. (B) Protein levels of cleaved PARP (C-PARP) and cleaved caspase-3 (C-CASP3) were evaluated by western blot analysis. GAPDH was used as a loading control. (C) Cell death was evaluated by TUNEL assay. (D) Huh7 cells were transfected with Flag-tagged SIRT6 or SIRT6 H133Y for $24 \mathrm{~h}$ and treated with DOX (1 $\mu$ M) for $36 \mathrm{~h}$. Protein expression of SIRT6, FOXO3, cleaved PARP and caspase-3 was evaluated by western blot analysis. (E) HepG2 cells were transfected with Flag-tagged SIRT6 or SIRT6 H133Y for $24 \mathrm{~h}$ and treated with DOX $(1 \mu \mathrm{M})$ for $36 \mathrm{~h}$. Cell death was evaluated by TUNEL assay. Data are presented as the mean \pm standard error of the mean of three independent experiments with technical duplicates. ${ }^{* *} \mathrm{P}<0.01,{ }^{* * *} \mathrm{P}<0.001$ vs. control; one- way ANOVA. PARP, poly(ADP ribose) polymerase; SIRT, sirtuin; FOXO3, forkhead box O3.

As indicated in Fig. 3C, at $24 \mathrm{~h}$ of doxorubicin treatment, FOXO3 binding to its target genes p27 and Bim was significantly increased. Consistent with this, RT-qPCR and western blotting results indicated that both mRNA and protein levels of p27 and Bim were significantly increased following treatment (Fig. 3D and E). In summary, these data demonstrated that doxorubicin treatment induces FOXO3 activation, which triggers expression of its target genes and results in cell cycle arrest and apoptosis.

SIRT6 interacts with FOXO3 and destabilizes FOXO3 by promoting ubiquitination. The aforementioned data indicated that SIRT6 is downregulated and FOXO3 is upregulated in response to doxorubicin. Therefore, it was assessed whether SIRT6 regulates FOXO3 protein stability. SIRT6 was overexpressed in HeLa cells and immunoprecipitation was performed to examine whether SIRT6 interacts with FOXO3. It was identified that these two proteins exhibit a direct interaction (Fig. 4A). Previous studies have suggested that multiple SIRT family members are able to deacetylate FOXO3 $(12,14,38)$. Thus, it was investigated whether SIRT6 deacetylates FOXO3. Flag-SIRT6 and SIRT6 H133Y were expressed in HeLa cells and FOXO3 proteins were immunoprecipitated. The acetylation level of FOXO3 was examined using a pan-acetyl Lysine antibody. It was identified that overexpression of SIRT6, but not the inactive form SIRT6 H133Y, significantly decreased the acetyl-FOXO3 level (Fig. 4B). It was also observed that SIRT6 significantly increased FOXO3 ubiquitination and the p-S253 FOXO3 level, which is critical for FOXO3 degradation (39) (Fig. 4B). These data demonstrated that SIRT6 interacts with FOXO3 and promotes its ubiquitination and degradation. Furthermore, it was investigated whether SIRT6 regulates FOXO3 protein stability in vitro. SIRT6 or SIRT6 H133Y was overexpressed in cells, and then the protein level of FOXO3 was evaluated by western blotting. SIRT6 overexpression itself was sufficient to increase p-FOXO3 S253 and reduce $\mathrm{FOXO} 3$ protein level, but this effect also required enzyme activity (Fig. 4C). The underlying mechanism of this may be that SIRT6 significantly decreases the FOXO3 protein half-life (Fig. 4D). In summary, these data indicated that SIRT6 interacts with FOXO3 and regulates FOXO3 protein stability by promoting its deacetylation and ubiquitination. 
A
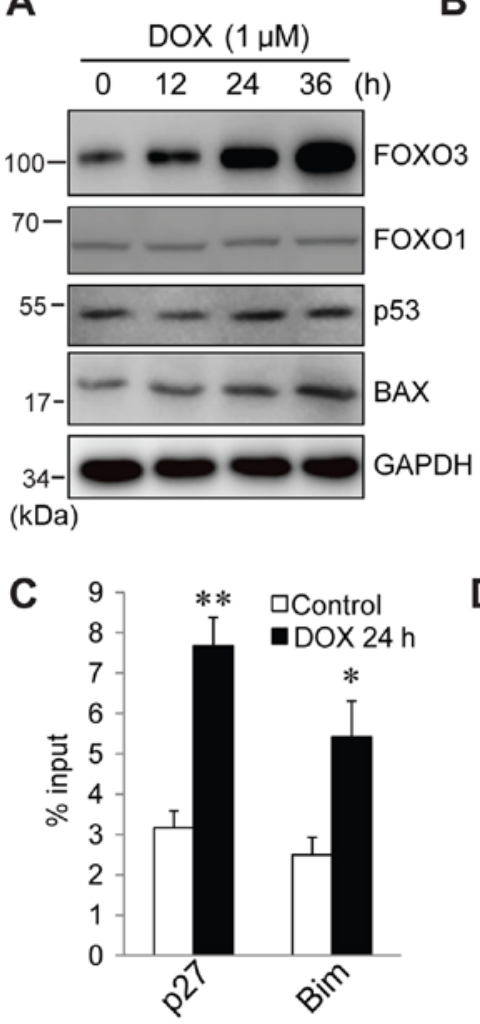

B

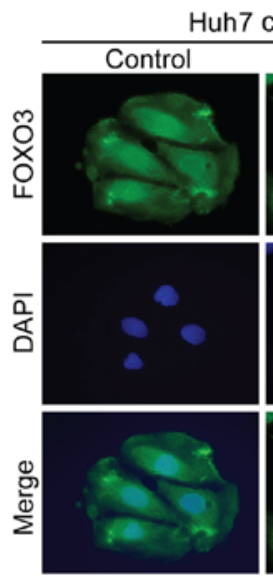

D

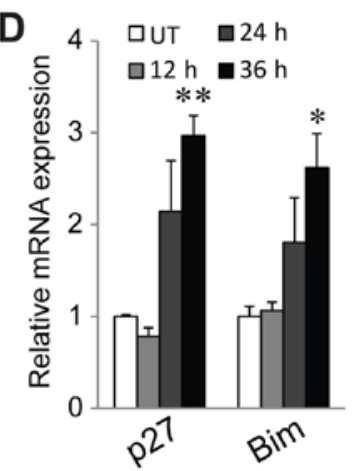

HepG2 cells
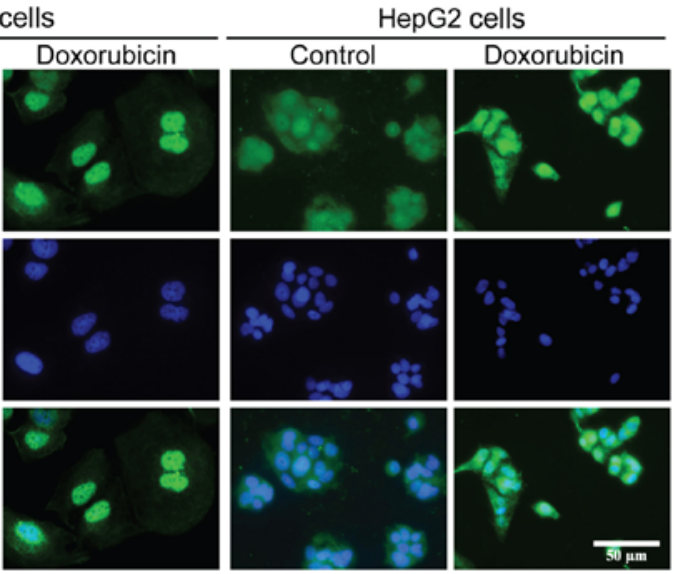

E

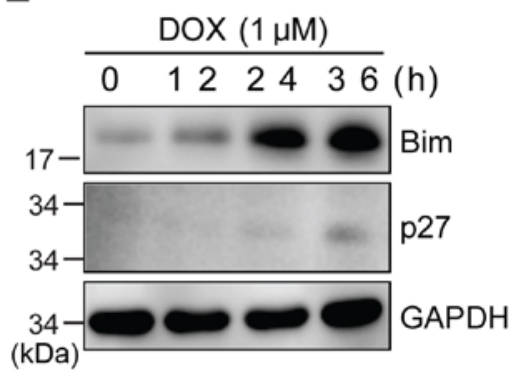

Figure 3. Doxorubicin (DOX) increases FOXO3 activation. HepG2 cells were treated with $1 \mu \mathrm{M}$ DOX for 36 h, then cells were harvested at various times as indicated. (A) Protein levels of FOXO3, FOXO1, p53 and Bax were evaluated by western blot analysis. GAPDH was used as a loading control. (B) Immunofluorescence staining of HepG2 and Huh7 cells that were either untreated (Control) or treated with DOX for various times as indicated by using FOXO3 antibody (green) and DAPI staining (blue). Scale bar, $50 \mu \mathrm{m}$. (C-E) HepG2 cells were either untreated (Control) or treated with DOX for $24 \mathrm{~h}$. (C) Promoter binding of FOXO3 was evaluated by chromatin immunoprecipitation assay. Data are presented as the mean \pm standard error of the mean of three independent experiments with technical duplicates. ${ }^{*} \mathrm{P}<0.05,{ }^{* *} \mathrm{P}<0.01$ vs. control, two-tailed unpaired Student's t-test. (D) p27 and Bim mRNA levels were evaluated by reverse transcription-quantitative polymerase chain reaction. (E) Protein levels were evaluated by western blot analysis. Graphs show mean \pm SEM of three independent experiments with technical duplicates. Data are presented as the mean \pm standard error of the mean of three independent experiments with technical duplicates. ${ }^{*} \mathrm{P}<0.05,{ }^{* *} \mathrm{P}<0.01$ vs. control; one-way ANOVA. FOXO3, forkhead box O3; FOXO1, forkhead box O1.

SIRT6 regulates doxorubicin-induced apoptosis through FOXO3. To examine whether FOXO3 is required for doxorubicin-induced apoptosis; two different lentiviral shFOXO3 transfections were used to knock down FOXO3 protein, and then cells were treated with doxorubicin. Western blotting results indicated that shFOXO3 transfection generated $>90 \%$ knockdown efficiency and loss of FOXO3 did not have any effects on SIRT6 expression (Fig. 5A). A TUNEL assay indicated that absence of FOXO3 significantly abolished doxorubicin-induced apoptosis (Fig. 5B). In order to evaluate whether SIRT6 regulates doxorubicin-induced apoptosis through FOXO3, it was first examined whether SIRT6 blocks nuclear accumulation of $\mathrm{FOXO} 3$ in response to doxorubicin. Cells were transfected with HA-FOXO3 or co-transfected with Flag-SIRT6. Following treatment with doxorubicin, cellular localization of FOXO3 was examined by immunofluorescence. As indicated in Fig. 2B, 24 h of doxorubicin treatment significantly induced nuclear localization of FOXO3 (Fig. 5C) which could be blocked by SIRT6 (Fig. 5D). Finally, we evaluated whether the blocking of doxorubicin-induced apoptosis by overexpression of SIRT6 was via FOXO3. SIRT6 and inactive SIRT6 H133Y were overexpressed in FOXO3-deficient cells followed by treatment with doxorubicin. SIRT6 and SIRT6 H133Y failed to block doxorubicin-induced apoptosis, as indicated by similar levels of cleaved PARP and cleaved caspase-3 (Fig. 5E) and TUNEL-positive cells (Fig. 5F) as compared with control cells. In summary, these data indicated that in response to doxorubicin treatment, SIRT6 is a critical factor that regulates FOXO3 nuclear localization, which is essential for inducing liver cancer cell death.

\section{Discussion}

In the present study, it was demonstrated that histone deacetylase SIRT6 is a key factor controlling doxorubicin-induced liver cancer cell death by modulating transcriptional factor FOXO3. In the absence of doxorubicin treatment, SIRT6 and FOXO3 interact with each other, and this interaction promotes FOXO3 deacetylation, ubiquitination and degradation. Following doxorubicin treatment, SIRT6 is downregulated, which in turn activates FOXO3 translocation into the nucleus and binding to its target genes p27 and Bim to cause cell death. Overexpression of SIRT6 abolishes FOXO3 activation and cell death following doxorubicin treatment. Finally, it was demonstrated that SIRT6 regulates doxorubicin-induced cell death through FOXO3; in the absence of FOXO3, SIRT6 overexpression is not able to prevent cell death. 
A
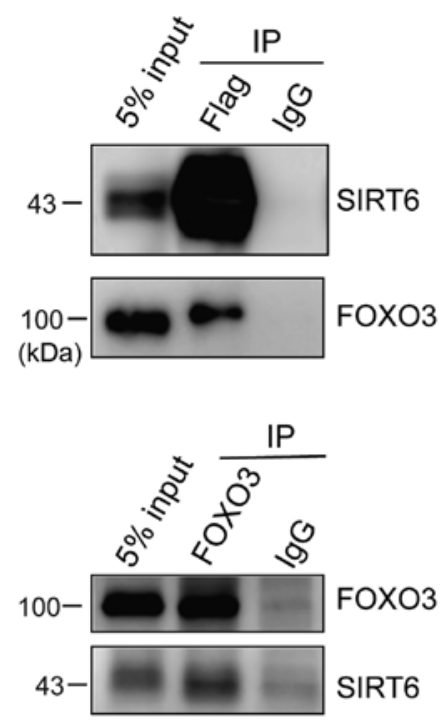

B

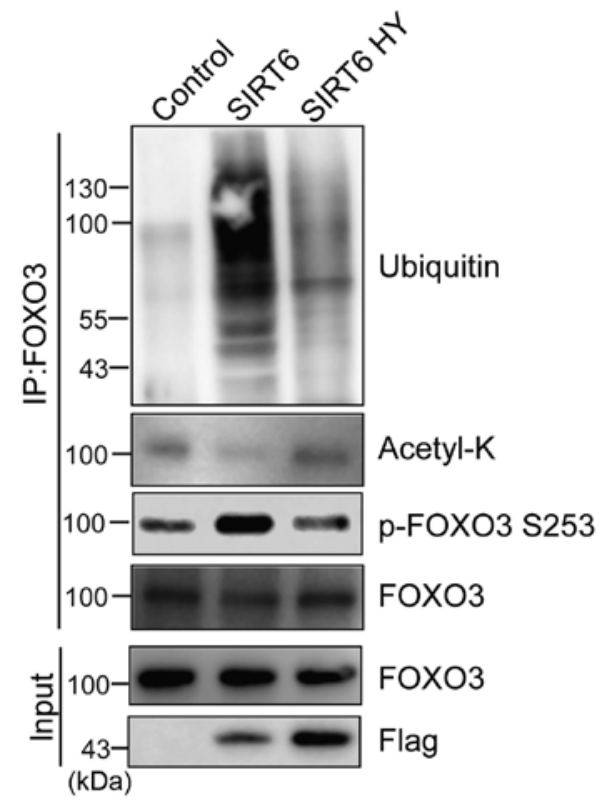

D

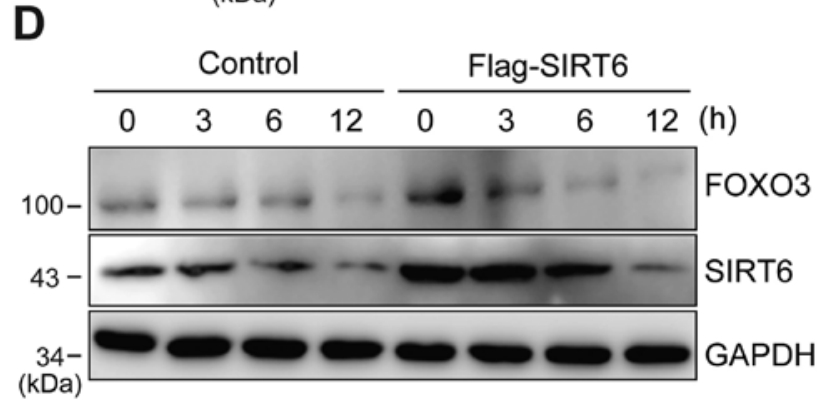

(
C

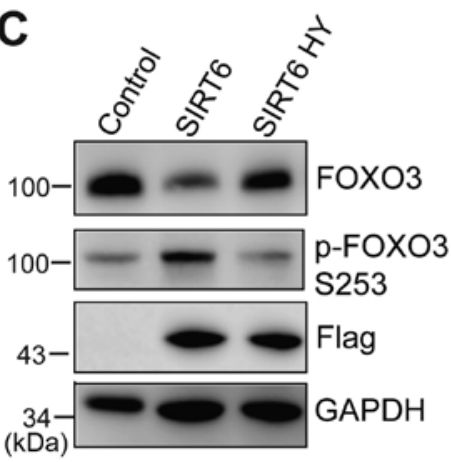

Figure 4. SIRT6 destabilizes FOXO3 by promoting FOXO3 ubiquitination. (A) Interaction between SIRT6 and FOXO3. HeLa cells were transfected with Flag-tagged SIRT6 for $24 \mathrm{~h}$. Flag-SIRT6 or FOXO3 were immunoprecipitated and the input and immune complexes were assessed for the presence of FOXO3 or SIRT6 by western blot analysis. (B) FOXO3 was immunoprecipitated from cell lysis as in (A) Acetylated, phosphorylated and ubiquitinated FOXO3 and total amount of FOXO3 were assessed by western blot analysis. (C) HepG2 cells were transfected with Flag-SIRT6 or SIRT6 H133Y for 24 h. Protein level of total FOXO3 and p-FOXO3 S253 were evaluated by western blot analysis. (D) HepG2 cells were transfected with Flag-SIRT6 for 24 h and cells were treated with CHX (100 $\mu \mathrm{M})$ for various times as indicated. Protein levels of FOXO3 and SIRT6 were evaluated by western blot analysis. FOXO3, forkhead box O3; SIRT, sirtuin; CHX, cycloheximide.

SIRT proteins are regulated dynamically by different stimuli. SIRT6 has long been recognized as an environmental nutrition sensor; in cases of nutrition restriction, SIRT6 is rapidly upregulated in a p53-dependent manner (40). Following LPS treatment, SIRTs are observed to be downregulated (23). While SIRT1 primarily increases degradation processes via post-translational mechanisms, SIRT7 decreases both transcription and protein stability (23). SIRT1 contains several post-translational modification sites that regulate protein stability and enzyme activity (41). In this study we have demonstrated that doxorubicin treatment results in a significant decrease in SIRT6 protein levels, which is critical for cell death. However, the mechanisms underlying SIRT6 downregulation require further investigation. It has been indicated that covalent modification of SIRT1 by oxidants/aldehydes results in a decrease in its enzymatic activity an increase in its degradation (42). There are multiple serine phosphorylation sites on SIRT1 (Ser27, Ser47, Ser659 and Ser661), which are regulated by diverse protein kinases (43-45). It has been demonstrated that JNK1 phosphorylation of SIRT1 at the aforementioned sites leads to proteasome-mediated degradation $(46,47)$. JNK has also been identified to phosphorylate SIRT6, but it is unclear whether this causes SIRT6 degradation (48). Notably, doxorubicin treatment induces JNK activation in multiple cancer types $(48,49)$.

FOXO3 is one of the most well-studied transcription factors and its activity is tightly regulated by post-transcriptional modification, including phosphorylation, ubiquitination, arginine methylation and acetylation (50). It is well documented that Akt-dependent phosphorylation is the major pathway that controls FOXO3 activity by promoting nuclear exclusion and degradation (51). Multiple SIRT proteins have been reported to deacetylate FOXO3 and regulate its transcriptional activity, including SIRT1, SIRT2, SIRT3, SIRT6 and SIRT7 $(13,14,19,23,51)$. In the present study, it was demonstrated that FOXO3 is also an SIRT6 target in liver cancer cells. SIRT6 interacts with FOXO3; overexpression of SIRT6, but not the enzyme-inactivated mutant, reduces FOXO3 protein half-life and redundancy, suggesting that SIRT6 regulates FOXO3 through deacetylation. Consistent with this, it was also identified that SIRT6 is able to reduce the acetylation level of FOXO3. The mechanism by which the FOXO3 
A
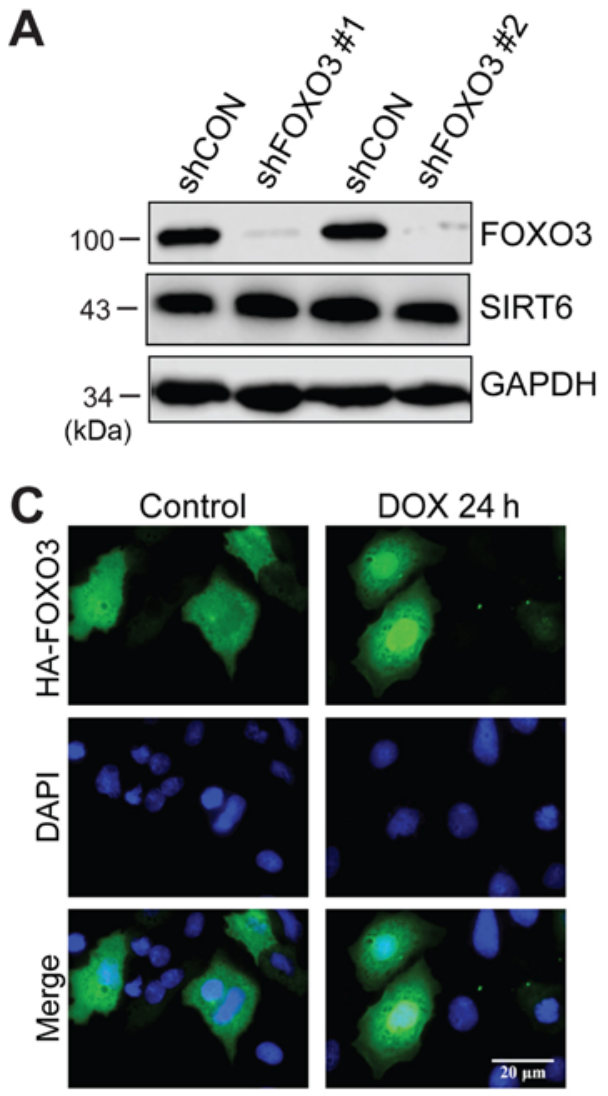

E

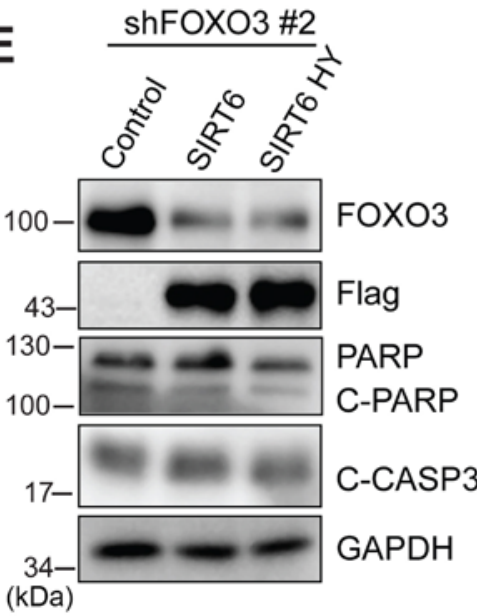

B
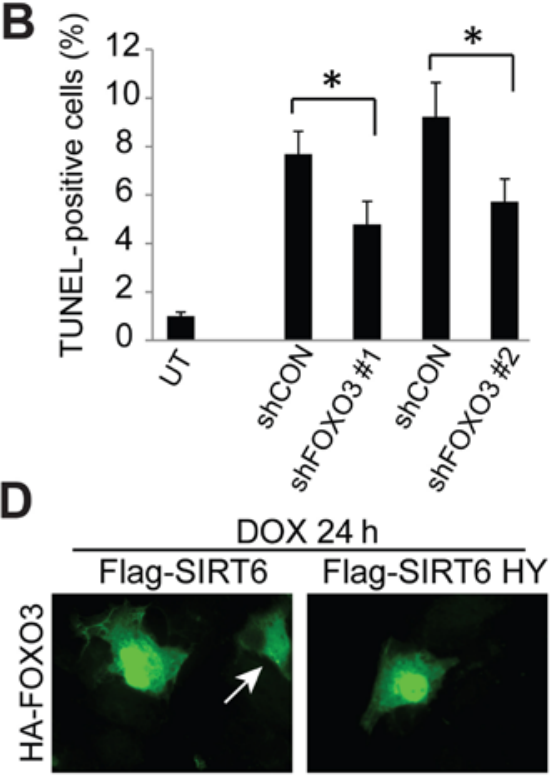

DOX $24 \mathrm{~h}$
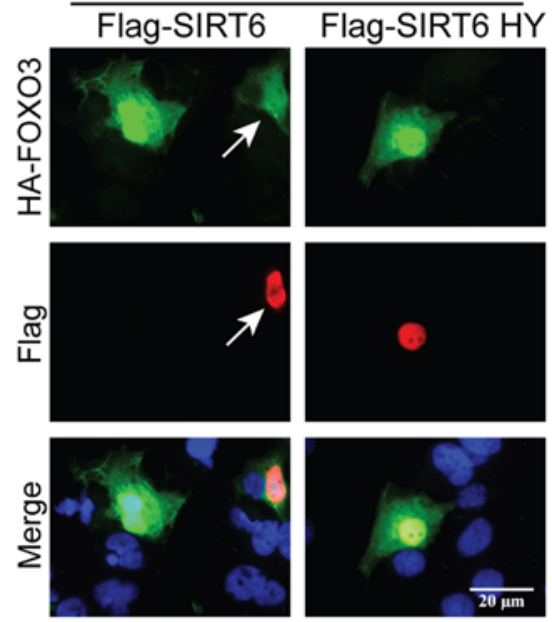

$\mathbf{F}$

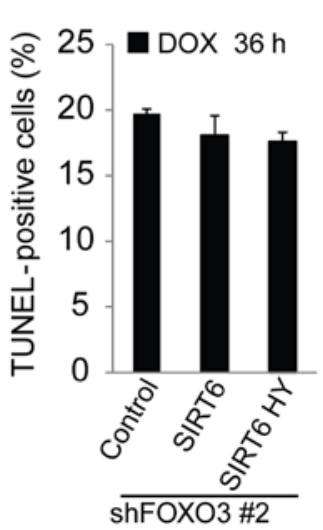

Figure 5. SIRT6 directs doxorubicin (DOX)-induced cell death through FOXO3. (A) HepG2 cells were transduced with shRNA specific for FOXO3 (shFOXO3) or non-targeting shRNA (shCON) for $72 \mathrm{~h}$. FOXO3 and SIRT6 protein levels were evaluated by western blot analysis. (B) Cells in A were treated with DOX for $36 \mathrm{~h}$ and cell death was evaluated by TUNEL assay. (C and D) HA-FOXO3 and/or Flag-SIRT6 were transfected into HepG2 cells followed by treatment with DOX for $24 \mathrm{~h}$. Immunofluorescence staining for FOXO3 and SIRT6 was performed using FOXO3 (green) and Flag (red) antibodies. Scale bar, $20 \mu \mathrm{m}$. (E and F) Cells in A were transfected with Flag-SIRT6 or SIRT6 H133Y for $24 \mathrm{~h}$ and treated with DOX for $36 \mathrm{~h}$. (E) Protein levels were evaluated by western blot analysis. (F) Cell death was evaluated by TUNEL assay. Data are presented as the mean \pm standard error of the mean of three independent experiments. ${ }^{*} \mathrm{P}<0.05$ vs. shCON; one-way ANOVA.

acetylation state controls FOXO3 stability is not clear, but it is reported that acetylation promotes the binding of FOXO3 to JNK1 (23). Thus, it is speculated that this may also increase FOXO3 binding to Akt, which triggers its phosphorylation and degradation. Notably, it was observed that SIRT6 overexpression increases p-FOXO3 S253 levels, which are critical for FOXO3 stability. A recent study also indicated that $\mathrm{p} 38$ phosphorylates FOXO3 and promotes its nuclear translocation in response to doxorubicin; it is not clear whether SIRT6 could block p38-induced FOXO3 phosphorylation.
The ability of SIRT6 and FOXO3 to regulate doxorubicin-dependent cell death raises the possibility that manipulation of this system may have therapeutic implications. Manipulating SIRT has long been demonstrated to have beneficial effects in various diseases. Resveratrol, a well-established SIRT activator, is recognized as an anti-apoptosis and anti-inflammatory compound that possesses beneficial effects in various diseases, including rheumatoid arthritis and type I diabetes $(52,53)$. SIRT inhibitor has been demonstrated to induce cancer cell apoptosis by increasing 
p53 acetylation $(53,54)$ and Myc degradation (55). The current data suggest that downregulation of SIRT6 is essential for doxorubicin-induced cancer cell death. Therefore, it is speculated that high expression of SIRT6 or failure to downregulate SIRT6 following doxorubicin treatment may contribute to TACE resistance. SIRT6 expression levels in human HCC tissues remain unclear due to conflicting reports, suggesting that SIRT6 is highly disease stage or population dependent, but it would also be interesting to investigate whether tumors with higher SIRT6 expression are also TACE resistant.

The present study demonstrated that downregulation of SIRT6 is required for doxorubicin-induced liver cancer cell death since it promotes FOXO3 nuclear localization and activation, leading to cell death. The current data also identified that SIRT6 controls FOXO3 by deacetylation, which is a novel mechanism. Furthermore, acetylation affects kinase binding to FOXO3 and whether there are apoptotic effects of FOXO3 that are independent of SIRT6 downregulation. To gain further understanding, these mechanisms should be investigated in a future study. Nonetheless, the current findings regarding the interaction between SIRT6 and FOXO3 elucidate a novel molecular mechanism by which SIRT proteins regulate cell function and point to SIRT6 as a potentially important target for liver cancer treatment.

\section{Acknowledgements}

We thank Dr Yi-Ming Tao for providing reagents for this study and his kind help in revising the manuscript.

\section{Funding}

The present study was supported in part by grants from the National Natural Science Foundation of China (grant no. 81473617), the Hunan Natural Science Foundation of China (grant no. 13JJ2032) and The Innovation Platform Open Foundation of Hunan Educational office (grant no. 16K066).

\section{Availability of data and materials}

The datasets used during the present study are available from the corresponding author upon reasonable request.

\section{Authors' contributions}

The manuscript was written through contributions of all authors. XFT conceived and designed the study. JQH, FD, $\mathrm{XPH}, \mathrm{WZ}$ and $\mathrm{XCZ}$ performed the experiments. XFT and JQH reviewed and edited the manuscript. All authors read and approved the manuscript and agree to be accountable for all aspects of the research in ensuring that the accuracy or integrity of any part of the work are appropriately investigated and resolved.

\section{Ethics approval and consent to participate}

All experimental protocols were approved by the Institutional Review Board of the Department of Laboratory Animal Science of Hunan University of Chinese Medicine (Changsha, China).

\section{Patient consent for publication}

Not applicable.

\section{Competing interests}

The authors declare that they have no competing interests.

\section{References}

1. Wong MC, Jiang JY, Goggins WB, Liang M, Fang Y, Fung FD, Leung $\mathrm{C}$, Wang $\mathrm{HH}$, Wong GL, Wong VW, et al: International incidence and mortality trends of liver cancer: A global profile. Sci Rep 7: 45846, 2017.

2. Altekruse SF, McGlynn KA and Reichman ME: Hepatocellular carcinoma incidence, mortality, and survival trends in the United States from 1975 to 2005. J Clin Oncol 27: 1485-1491, 2009.

3. Hadzic N and Finegold MJ: Liver neoplasia in children. Clin Liver Dis 15: 443-462, vii-x, 2011.

4. Horton JD, Lee S, Brown SR, Bader J and Meier DE: Survival trends in children with hepatoblastoma. Pediatr Surg Int 25: 407-412, 2009.

5. Otte JB: Progress in the surgical treatment of malignant liver tumors in children. Cancer Treat Rev 36: 360-371, 2010.

6. Davis GL, Dempster J, Meler JD, Orr DW, Walberg MW, Brown B, Berger BD, O'Connor JK and Goldstein RM: Hepatocellular carcinoma: Management of an increasingly common problem. Proc 21: 266-280, 2008

7. Dhanasekaran R, Limaye A and Cabrera R: Hepatocellular carcinoma: Current trends in worldwide epidemiology, risk factors, diagnosis, and therapeutics. Hepat Med 4: 19-37, 2012.

8. Cox J and Weinman S: Mechanisms of doxorubicin resistance in hepatocellular carcinoma. Hepat Oncol 3: 57-59, 2016.

9. North BJ and Verdin E: Sirtuins: Sir2-related NAD-dependent protein deacetylases. Genome Biol 5: 224, 2004.

10. Canto C, Gerhart-Hines Z, Feige JN, Lagouge M, Noriega L, Milne JC, Elliott PJ, Puigserver P and Auwerx J: AMPK regulates energy expenditure by modulating $\mathrm{NAD}^{+}$metabolism and SIRT1 activity. Nature 458: 1056-1060, 2009.

11. Satoh A, Brace CS, Rensing N, Cliften P, Wozniak DF, Herzog ED, Yamada KA and Imai S: Sirtl extends life span and delays aging in mice through the regulation of $\mathrm{Nk} 2$ homeobox 1 in the DMH and LH. Cell Metab 18: 416-430, 2013.

12. Wang Y, Zhu Y, Xing S, Ma P and Lin D: SIRT 5 prevents cigarette smoke extract-induced apoptosis in lung epithelial cells via deacetylation of $\mathrm{FOXO}_{3}$. Cell Stress Chaperones 20: 805-810, 2015.

13. Tseng AH, Shieh SS and Wang DL: SIRT3 deacetylates FOXO3 to protect mitochondria against oxidative damage. Free Radic Biol Med 63: 222-234, 2013.

14. Brunet A, Sweeney LB, Sturgill JF, Chua KF, Greer PL, Lin Y, Tran H, Ross SE, Mostoslavsky R, Cohen HY, et al: Stress-dependent regulation of FOXO transcription factors by the SIRT1 deacetylase. Science 303: 2011-2015, 2004.

15. Simic P, Zainabadi K, Bell E, Sykes DB, Saez B, Lotinun S, Baron R, Scadden D, Schipani E and Guarente L: SIRT1 regulates differentiation of mesenchymal stem cells by deacetylating $\beta$-catenin. EMBO Mol Med 5: 430-440, 2013.

16. Jeong J, Juhn K, Lee H, Kim SH, Min BH, Lee KM, Cho MH, Park GH and Lee KH: SIRT1 promotes DNA repair activity and deacetylation of Ku70. Exp Mol Med 39: 8-13, 2007.

17. Marquardt JU, Fischer K, Baus K, Kashyap A, Ma S, Krupp M, Linke M, Teufel A, Zechner U, Strand D, et al: Sirtuin-6-dependent genetic and epigenetic alterations are associated with poor clinical outcome in hepatocellular carcinoma patients. Hepatology 58: 1054-1064, 2013

18. Ponugoti B, Kim DH, Xiao Z, Smith Z, Miao J, Zang M, Wu SY, Chiang CM, Veenstra TD and Kemper JK: SIRT1 deacetylates and inhibits SREBP-1C activity in regulation of hepatic lipid metabolism. J Biol Chem 285: 33959-33970, 2010.

19. Wang F, Nguyen M, Qin FX and Tong Q: SIRT2 deacetylates FOXO3a in response to oxidative stress and caloric restriction. Aging Cell 6: 505-514, 2007.

20. Jin YH, Kim YJ, Kim DW, Baek KH, Kang BY, Yeo CY and Lee KY: Sirt2 interacts with 14-3-3 beta/gamma and down-regulates the activity of p53. Biochem Biophys Res Commun 368: 690-695, 2008 . 
21. Rothgiesser KM, Erener S, Waibel S, Luscher B and Hottiger MO: SIRT2 regulates NF- $\kappa \mathrm{B}$ dependent gene expression through deacetylation of p65 Lys310. J Cell Sci 123: 4251-4258, 2010

22. Vaziri H, Dessain SK, Ng Eaton E, Imai SI, Frye RA, Pandita TK, Guarente L and Weinberg RA: hSIR2SIRT1 functions as an NAD-dependent p53 deacetylase. Cell 107: 149-159, 2001.

23. Li Z, Bridges B, Olson J and Weinman SA: The interaction between acetylation and serine-574 phosphorylation regulates the apoptotic function of FOXO3. Oncogene 36: 1887-1898, 2017.

24. Paredes S, Villanova L and Chua KF: Molecular pathways: Emerging roles of mammalian sirtuin SIRT7 in cancer. Clin Cancer Res 20: 1741-1746, 2014.

25. Kaidi A, Weinert BT, Choudhary C and Jackson SP: Human SIRT6 promotes DNA end resection through CtIP deacetylation. Science 329: 1348-1353, 2010.

26. Kim HS, Xiao C, Wang RH, Lahusen T, Xu X, Vassilopoulos A, Vazquez-Ortiz G, Jeong WI,Park O, Ki SH, et al: Hepatic-specific disruption of SIRT6 in mice results in fatty liver formation due to enhanced glycolysis and triglyceride synthesis. Cell Metab 12: 224-236, 2010.

27. Xiao C, Kim HS, Lahusen T, Wang RH, Xu X, Gavrilova O, Jou W, Gius D and Deng CX: SIRT6 deficiency results in severe hypoglycemia by enhancing both basal and insulin-stimulated glucose uptake in mice. J Biol Chem 285: 36776-36784, 2010.

28. Zhong L, D'Urso A, Toiber D, Sebastian C, Henry RE, Vadysirisack DD, Guimaraes A, Marinelli B, Wikstrom JD, Nir T, et al: The histone deacetylase Sirt6 regulates glucose homeostasis via Hiflalpha. Cell 140: 280-293, 2010.

29. Kugel S, Sebastian C, Fitamant J, Ross KN, Saha SK, Jain E, Gladden A, Arora KS, Kato Y, Rivera MN, et al: SIRT6 suppresses pancreatic cancer through control of Lin28b. Cell 165: 1401-1415, 2016.

30. Ioris RM, Galie M, Ramadori G, Anderson JG, Charollais A, Konstantinidou G, Brenachot X, Aras E, Goga A, Ceglia N, et al: SIRT6 suppresses cancer stem-like capacity in tumors with PI3K activation independently of its deacetylase activity. Cell Rep 18 1858-1868, 2017

31. Sebastian C, Zwaans BM, Silberman DM, Gymrek M, Goren A, Zhong L, Ram O, Truelove J, Guimaraes AR, Toiber D, et al: The histone deacetylase SIRT6 is a tumor suppressor that controls cancer metabolism. Cell 151: 1185-1199, 2012.

32. Lee N, Ryu HG, Kwon JH, Kim DK, Kim SR, Wang HJ, Kim KT and Choi KY: SIRT6 depletion suppresses tumor growth by promoting cellular senescence induced by DNA damage in HCC. PLoS One 11: e0165835, 2016.

33. Ran LK, Chen Y, Zhang ZZ, Tao NN, Ren JH, Zhou L, Tang H, Chen X, Chen K, Li WY, et al: SIRT6 overexpression potentiates apoptosis evasion in hepatocellular carcinoma via BCL2-associated X protein-dependent apoptotic pathway. Clin Cancer Res 22: 3372-3382, 2016.

34. Li Z, Zhao J, Tikkhanovich I, Kuravi S, Helzberd J, Dorko K, Roberts B, Kumer S and Weinman SA: Serine 574 phosphorylation alters transcriptional programming of $\mathrm{FOXO} 3$ by selectively enhancing apoptotic gene expression. Cell Death Differ 23 . 583-595, 2016

35. Zhang P, Tu B, Wang H, Cao Z, Tang M, Zhang C, Gu B, Li Z, Wang L, Yang Y, et al: Tumor suppressor p53 cooperates with SIRT6 to regulate gluconeogenesis by promoting foxO1 nuclear exclusion. Proc Natl Acad Sci U S A 111: 10684-10689, 2014

36. Tao R, Xiong X, DePinho RA, Deng CX and Dong XC: FoxO3 transcription factor and Sirt6 deacetylase regulate low density lipoprotein (LDL)-cholesterol homeostasis via control of the proprotein convertase subtilisin/kexin type 9 (Pcsk9) gene expression. J Biol Chem 288: 29252-29259, 2013.

37. Song MY, Wang J, Ka SO, Bae EJ and Park BH: Insulin secretion impairment in Sirt6 knockout pancreatic beta cells is mediated by suppression of the FoxO1-Pdx1-Glut2 pathway. Sci Rep 6 : 30321, 2016.

38. Wang F, Chan $\mathrm{CH}$, Chen K, Guan X, Lin HK and Tong Q: Deacetylation of FOXO3 by SIRT1 or SIRT2 leads to Skp2-mediated FOXO3 ubiquitination and degradation. Oncogene 31: 1546-1557, 2012.
39. Dobson M, Ramakrishnan G, Ma S, Kaplun L, Balan V, Fridman R and Tzivion G: Bimodal regulation of FoxO3 by AKT and 14-3-3. Biochim Biophys Acta 1813: 1453-1464, 2011.

40. Kanfi Y, Shalman R, Peshti V, Pilosof SN, Gozlan YM, Pearson KJ, Lerrer B, Moazed D, Marine JC, de Cabo R, et al: Regulation of SIRT6 protein levels by nutrient availability. FEBS Lett 582: 543-548, 2008.

41. Flick F and Luscher B: Regulation of sirtuin function by posttranslational modifications. Front Pharmacol 3: 29, 2012.

42. Caito S, Rajendrasozhan S, Cook S, Chung S, Yao H, Friedman AE, Brookes PS and Rahman I: SIRT1 is a redox-sensitive deacetylase that is post-translationally modified by oxidants and carbonyl stress. FASEB J 24: 3145-3159, 2010.

43. Sasaki T, Maier B, Koclega KD, Chruszcz M, Gluba W, Stukenberg PT, Minor W and Scrable H: Phosphorylation regulates SIRT1 function. PLoS One 3: e4020, 2008.

44. Zschoernig B and Mahlknecht U: Carboxy-terminal phosphorylation of SIRT1 by protein kinase CK2. Biochem Biophys Res Commun 381: 372-377, 2009.

45. Olsen JV, Blagoev B, Gnad F, Macek B, Kumar C, Mortensen P and Mann M: Global, in vivo, and site-specific phosphorylation dynamics in signaling networks. Cell 127: 635-648, 2006.

46. Nasrin N, Kaushik VK, Fortier E, Wall D, Pearson KJ, de Cabo R and Bordone L: JNK1 phosphorylates SIRT1 and promotes its enzymatic activity. PLoS One 4: e8414, 2009.

47. Gao Z, Zhang J, Kheterpal I, Kennedy N, Davis RJ and Ye J: Sirtuin 1 (SIRT1) protein degradation in response to persistent c-Jun N-terminal kinase 1 (JNK1) activation contributes to hepatic steatosis in obesity. J Biol Chem 286: 22227-22234, 2011.

48. Van Meter M, Simon M, Tombline G, May A, Morello TD, Hubbard BP, Bredbenner K, Park R, Sinclair DA, Bohr VA, et al: JNK phosphorylates SIRT6 to stimulate DNA double-strand break repair in response to oxidative stress by recruiting PARP1 to DNA breaks. Cell Rep 16: 2641-2650, 2016.

49. Kim J and Freeman MR: JNK/SAPK mediates doxorubicin-induced differentiation and apoptosis in MCF-7 breast cancer cells. Breast Cancer Res Treat 79: 321-328, 2003.

50. Calnan DR and Brunet A: The foxo code. Oncogene 27: 2276-2288, 2008.

51. Khongkow M, Olmos $\mathrm{Y}$, Gong $\mathrm{C}$, Gomes AR, Monteiro LJ, Yagüe E, Cavaco TB, Khongkow P, Man EP, Laohasinnarong S, et al: SIRT6 modulates paclitaxel and epirubicin resistance and survival in breast cancer. Carcinogenesis 34: 1476-1486, 2013.

52. Elmali N, Baysal O, Harma A, Esenkaya I and Mizrak B: Effects of resveratrol in inflammatory arthritis. Inflammation 30: 1-6, 2007.

53. Lee SM, Yang H, Tartar DM, Gao B, Luo X, Ye SQ, Zaghouani H and Fang D: Prevention and treatment of diabetes with resveratrol in a non-obese mouse model of type 1 diabetes. Diabetologia 54: 1136-1146, 2011.

54. Peck B, Chen CY, Ho KK, Di Fruscia P, Myatt SS, Coombes RC, Fuchter MJ, Hsiao CD and Lam EW: SIRT inhibitors induce cell death and p53 acetylation through targeting both SIRT1 and SIRT2. Mol Cancer Ther 9: 844-855, 2010.

55. Jing H, Hu J, He B, Negrón Abril YL, Stupinski J, Weiser K, Carbonaro M, Chiang YL, Southard T, Giannakakou P, et al: A SIRT2-selective inhibitor promotes c-Myc oncoprotein degradation and exhibits broad anticancer activity. Cancer Cell 29: 297-310, 2016.

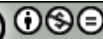

This work is licensed under a Creative Commons Attribution-NonCommercial-NoDerivatives 4.0 International (CC BY-NC-ND 4.0) License. 